\title{
Antimutagenic activity of probiotic drugs on the example of Drosophila
}

\author{
Evgenia Bochkareva, Kristina Degtyareva*, Olga Voloschenko and Olesya Makanina \\ Belgorod State National Research University, 308015, Belgorod, Russia
}

\begin{abstract}
Probiotic preparations were obtained on the basis of cryopreserved forms of pure cultures of $L$. plantarum and B. subtilis using freeze drying. The antimutagenic activity of the obtained probiotic preparations was investigated in relation to $1 \%, 0.1 \%$ and $0.01 \%$ concentrations of cobalt sulfate using the example of dominant lethal mutations of Drosophila. A positive antimutagenic effect of probiotic preparations was shown in relation to $0.1 \%$ and $0.01 \%$ concentrations of $\mathrm{CoSO}_{4}$ with an exposure duration of at least 3 days.

Keywords: probiotic drugs, Drosophia, antimutagenic activity.
\end{abstract}

\section{Introduction}

Probiotic drugs are a promising alternative to chemotherapeutic drugs, agrochemicals, pesticides, and antibiotics, which are potentially hazardous to the health of people and farm animals.

Chemicalization and intensification of industry and agriculture leads to an increase in the mutational load in populations of plants, animals, and humans, however, compensatory mechanisms in living beings that act against mutations are limited [1]. One of the approaches to reducing the destructive effect of chemical mutagens on organisms is to search for substances with antimutagenic properties.

Until the late 1980s, bacteria were hardly studied as sources of antimutagens. However, taking into account the general fundamental metabolic reactions of prokaryotes and eukaryotes, as well as the ability of bacteria to carry out reactions for the synthesis of substances with protective and reactivating properties, it can be assumed that bacteria can become a source of new and valuable antimutagens [2]. This assumption formed the basis for the relevance of our research.

The main goal was to identify the antimutagenic effect of dry probiotic preparations based on Lactobacillus plantarum and Bacillus subtilis cultures in relation to cobalt sulfate using the example of early and late embryonic lethal mutations in Drosophila.

The proposed hypothesis: experimental groups of Drosophila kept on a medium with probiotic preparations will have a lower proportion of embryonic lethal mutations in comparison with control groups kept under standard conditions.

Tasks:

1. Get freeze-dried probiotic preparations based on L. plantarum and B. Subtilis

\footnotetext{
*Correspondingauthor:degtyareva@bsu.edu.ru
} 
2. Check the presence or absence of antimutagenic activity of the obtained probiotic preparations in relation to various concentrations of cobalt sulfate.

\section{Material and methods}

The first stage of the work was to obtain dry probiotic preparations, which was carried out on the basis of cryopreserved forms of pure cultures of L. plantarum and B. subtilis. For this, microorganisms were inoculated first by the exhaust stroke method, and then by a continuous stroke on Petri dishes on LB medium, then the germinated colonies of pure cultures were separated and suspended in sterile distilled water and dried for 16 hours at a temperature $\left(-50^{\circ} \mathrm{C}\right)$ and a pressure of 0.05 mbar in a freeze dryer.

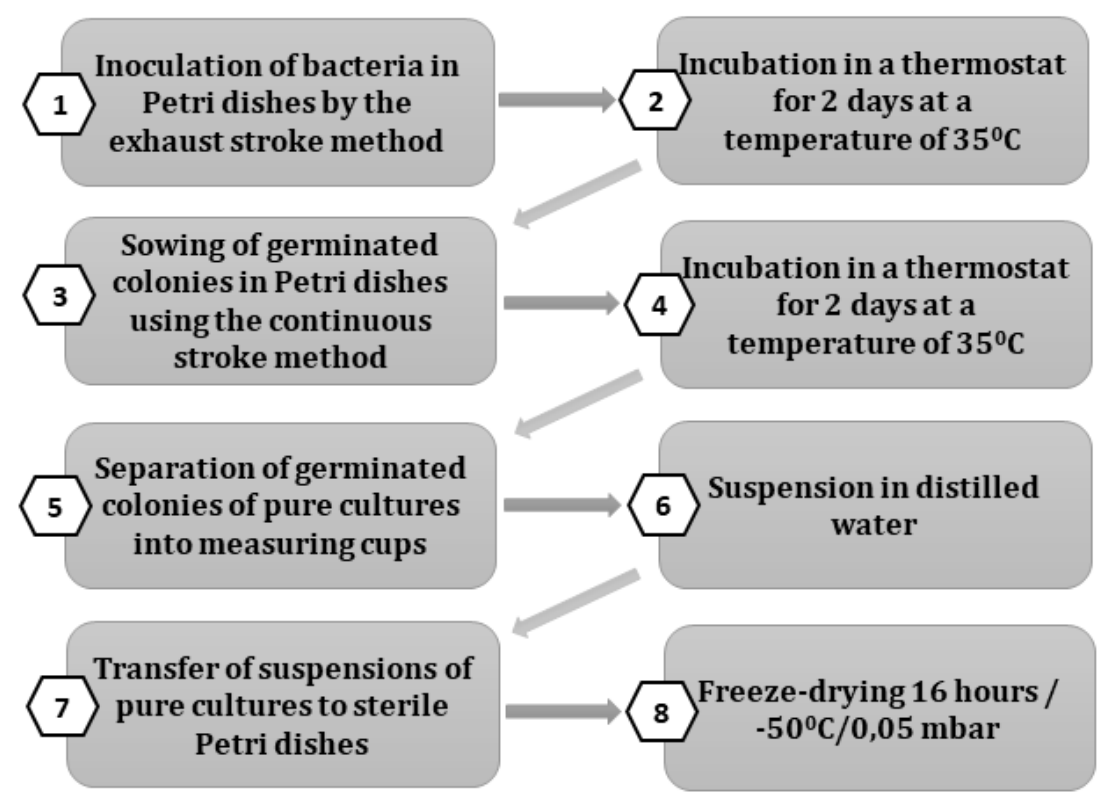

Fig. 1. Scheme for obtaining probiotic preparations

The obtained dry probiotic preparations were investigated for the presence of antimutagenic activity against cobalt sulfate at concentrations of $1 \%, 0.1 \%$ and $0.01 \%$ by taking into account the dominant lethal mutations (DLM) of Drosophila melanogaster wild type Canton-S from the laboratory collections of the Department of Biotechnology and Microbiology by the method of Belokon [3]. For this, the experimental groups of male Drosophila were kept on a nutrient medium with probiotics for 1, 3 and 7 days, after which they were exposed to the indicated concentrations of cobalt sulfate. First, stock solutions of salt were prepared, and then the required volume of stock solution was added to the nutrient medium for Drosophila to obtain the required concentrations. Then we set up crosses with virgid females on a standard medium for keeping flies. The embryonic mortality of F1 offspring and viability at all stages of ontogenetic development were determined by counting the number of undeveloped and developed embryos (the number of developed embryos was determined by the number of shells from eggs), the number of pupae and adults. The ratio of dead embryos to the total number of eggs, expressed as a percentage, was considered as DLM.

\section{Results and discussion}


Based on the data of counting the number of F1 offspring at all stages of ontogenetic development of Drosophila (egg, larva, numph, imago), the graphs of viability were constructed. The $\mathrm{Y}$-axis shows the viability, expressed as a percentage, and the $\mathrm{X}$-axis shows the stage of ontogeny. The total number of eggs laid by females was taken as $100 \%$, then the percentage of the remaining viable individuals at each stage of development was calculated proportionally ( $\mathrm{a}-\mathrm{F} 1$ offspring obtained from males kept on nutrient media with a probiotic for 1 day, $\mathrm{b}-3$ days, $\mathrm{c}-7$ days) (Fig. 2).

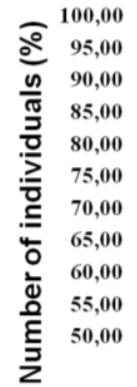

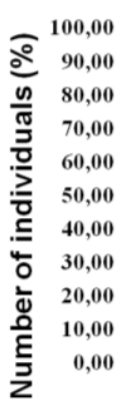

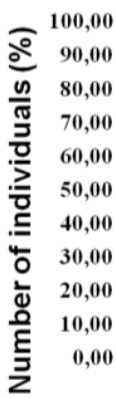

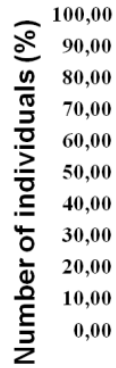

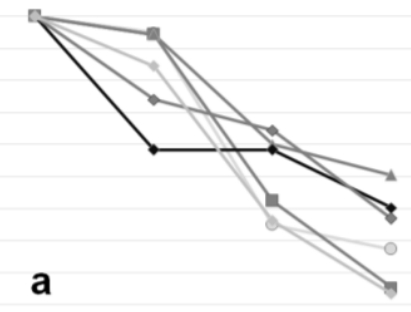

Egg Larva Nymph Imago

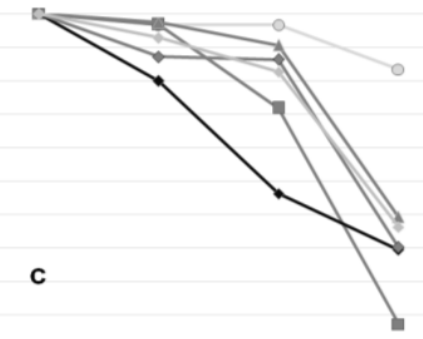

Egg Larva Nymph Imago

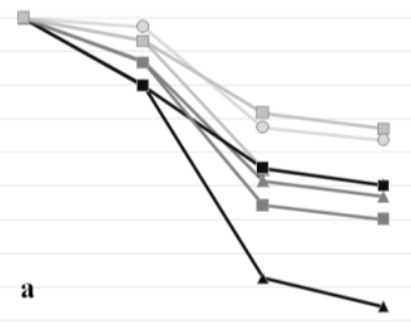

Egg Larva Nymph Imago

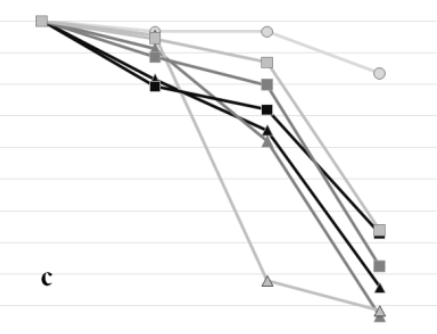

Egg Larva Nymph Imago

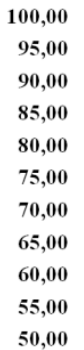

b

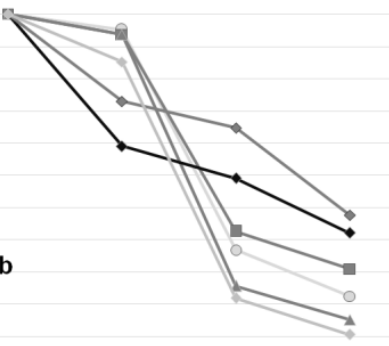

Egg Larva Nymph Imago

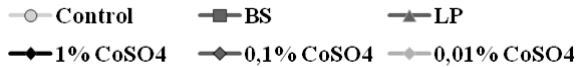

100,00

$\mathbf{9 0 , 0 0}$

80,00

$\mathbf{7 0 , 0 0}$

60,00

$\mathbf{5 0 , 0 0}$

40,00

30,00

20,00

10,00

0,00

Egg Larva Nymph Imago

Fig. 2. Dynamics of the number of viable F1 descendants at different stages of ontogenesis 
Analysis of the graphs showed that the greatest death of individuals in almost all samples is observed at the larval stage, with the exception of samples in which the offspring of males kept on probiotic preparations developed for 7 days. In these samples, the greatest death of individuals was observed at the pupal stage. It is paradoxical that even among the offspring from males in the control group (that is, those that were not exposed to heavy metal), there is death of individuals at the larval and pupal stage, while embryonic mortality in all control samples does not exceed $3-4 \%$. This fact may be due to the fact that for the most convenient counting of the number of eggs, a small amount of nutrient medium was added to the cups for setting up the crosses. Competition for a limited food resource could lead to the death of individuals at the larval and pupal stages. To test this hypothesis, a correlation analysis of the dependence of the number of individuals killed at the larval and pupal stage on the total number of viable eggs was carried out; however, no reliable linear relationship was found.

Figure 3 shows the results of counting the number of viable embryos and non-viable embryos, which are mistaken for dominant lethal mutations leading to the death of individuals. They are usually divided into early embryonic lethal mutations (EELM) and late embryonic lethal mutations (LELM), which can be quite clearly distinguished from each other. The early ones are milky-white undeveloped embryos, the late ones are brown. Such embryos have segregation stages (segmental structure) well visible under a microscope.

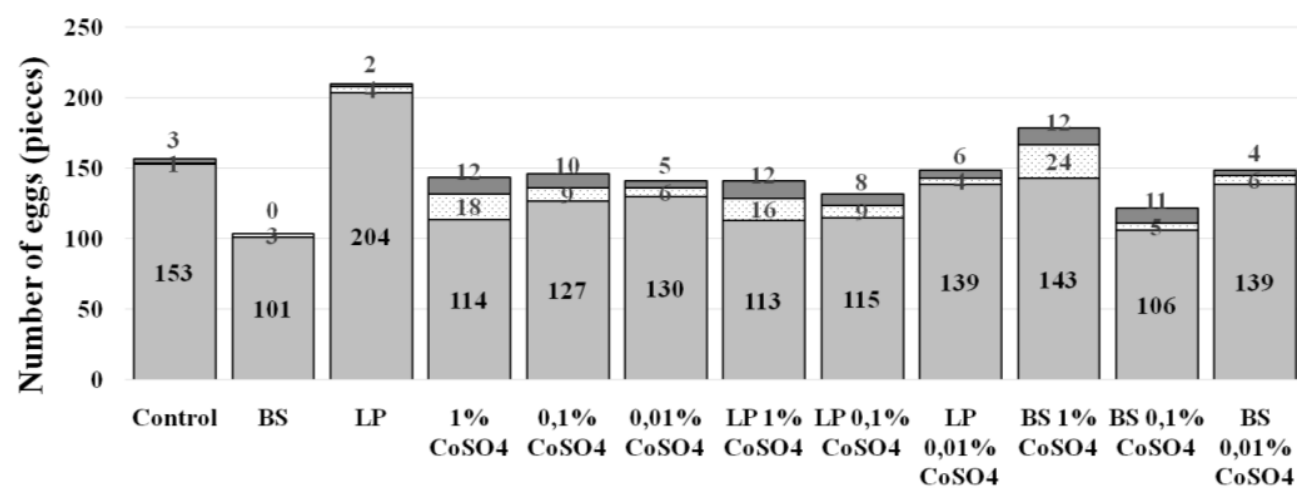

a

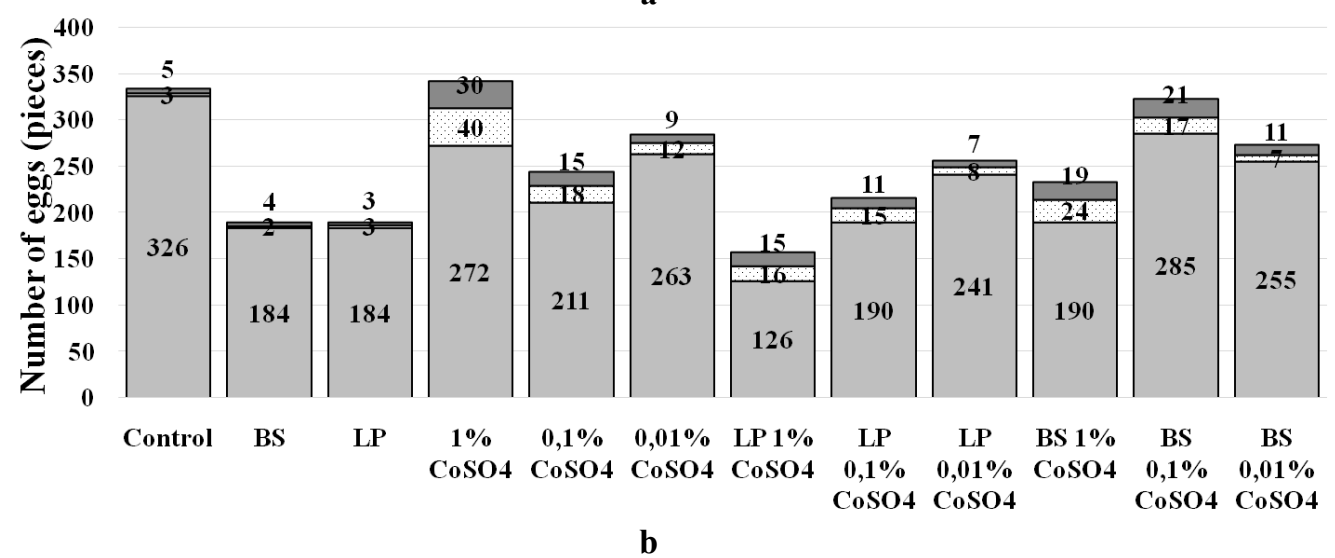




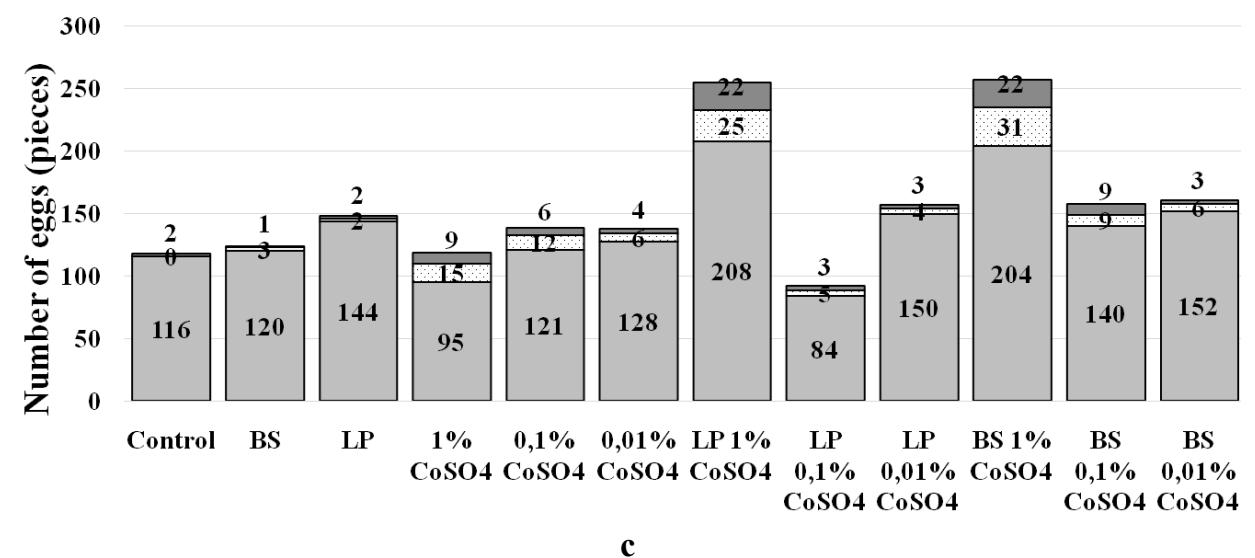

- Viable eggs

Early embryonic lethal mutations

- Late embryonic lethal mutations

Fig. 3. The number of viable eggs and early and late embryonic lethal samples

Table 1. The mean value of dominant lethal mutations in the sample

\begin{tabular}{|c|c|c|c|}
\hline \multirow{2}{*}{ Samples } & \multicolumn{3}{|c|}{ Mean DLM (\%) } \\
\cline { 2 - 4 } & $\mathbf{a}$ & $\mathbf{b}$ & $\mathbf{c}$ \\
\hline Control & $2,48 \pm 0,4$ & $2,42 \pm 0,32$ & $1,30 \pm 0,65$ \\
\hline $\mathrm{BS}$ & $3,21 \pm 0,73$ & $3,02 \pm 0,16$ & $3,34 \pm 0,35$ \\
\hline $\mathrm{LP}$ & $3,09 \pm 0,55$ & $3,03 \pm 0,5$ & $2,66 \pm 0,33$ \\
\hline $1 \% \mathrm{CoSO}_{4}$ & $21,05 \pm 0,91$ & $20,48 \pm 0,22$ & $20,09 \pm 0,09$ \\
\hline $0,1 \% \mathrm{CoSO}_{4}$ & $13,09 \pm 0,4$ & $13,14 \pm 0,7$ & $12,94 \pm 0,07$ \\
\hline $0,01 \% \mathrm{CoSO}_{4}$ & $7,82 \pm 0,29$ & $7,46 \pm 0,22$ & $7,05 \pm 0,24$ \\
\hline $\mathrm{LP} \rightarrow 1 \% \mathrm{CoSO}_{4}$ & $19,86 \pm 0,15$ & $19,72 \pm 0,16$ & $18,48 \pm 0,20$ \\
\hline $\mathrm{LP} \rightarrow 0,1 \% \mathrm{CoSO}_{4}$ & $12,87 \pm 0,35$ & $12,11 \pm 0,20$ & $8,47 \pm 0,32$ \\
\hline $\mathrm{LP} \rightarrow 0,01 \% \mathrm{CoSO}_{4}$ & $6,72 \pm 0,63$ & $5,96 \pm 0,21$ & $4,31 \pm 0,53$ \\
\hline $\mathrm{BS} \rightarrow 1 \% \mathrm{CoSO}_{4}$ & $20,27 \pm 0,59$ & $18,29 \pm 0,43$ & $20,61 \pm 0,77$ \\
\hline $\mathrm{BS} \rightarrow 0,1 \% \mathrm{CoSO}_{4}$ & $13,13 \pm 0,1$ & $11,74 \pm 0,14$ & $11,38 \pm 0,16$ \\
\hline $\mathrm{BS} \rightarrow 0,01 \% \mathrm{CoSO}_{4}$ & $6,82 \pm 0,54$ & $6,46 \pm 0,29$ & $5,60 \pm 0,12$ \\
\hline
\end{tabular}




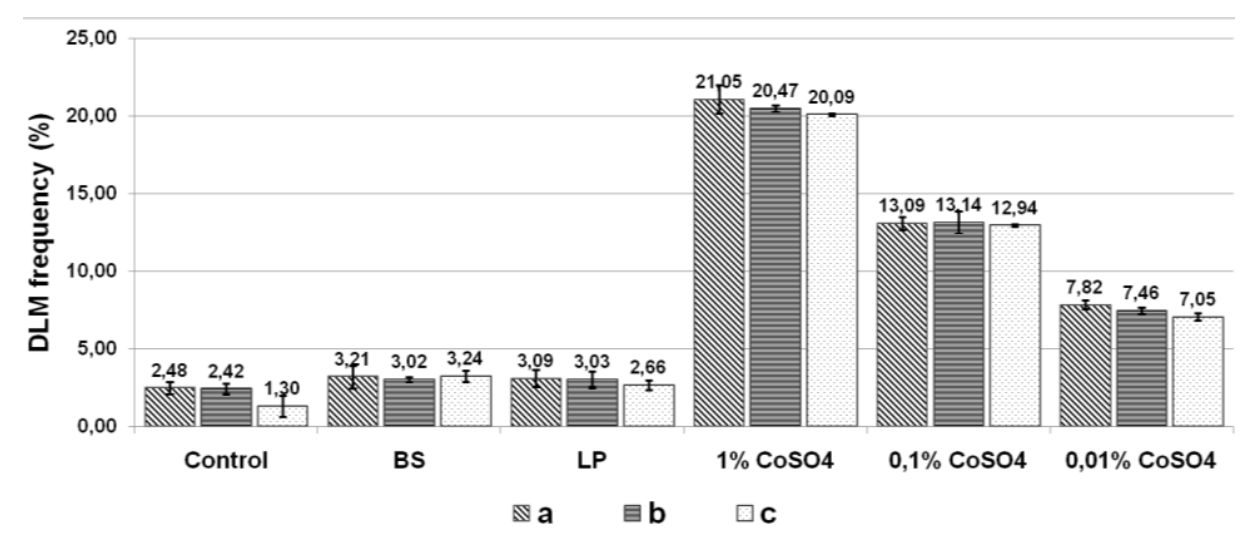

Fig. 4. Dynamics of death of F1 offspring at different stages of ontogenesis

First, to check the purity of the experiment, the frequency of DLM was compared in samples with the offspring of males in the control group and in samples with the offspring of males kept for 1, 3 and 7 days on media with probiotics, but not exposed to heavy metals. In all replicates, the absence of significant differences is observed, which may indicate the absence of mutagenic activity of the probiotic drugs themselves or metabolites secreted by bacteria. A similar comparison was made for samples with the offspring of males in the control group and samples with the offspring of males exposed to cobalt sulfate, but not previously contained on probiotics. All replicates show significant differences. This may indicate the presence of mutagenic activity of the heavy metal salt. Moreover, with an increase in concentration, the frequency of DLM increases.

Further, this indicator was analyzed in samples with the offspring of males, previously exposed to the probiotic preparations under study, and then exposed to cobalt sulfate (data are shown in Figures 5-7)

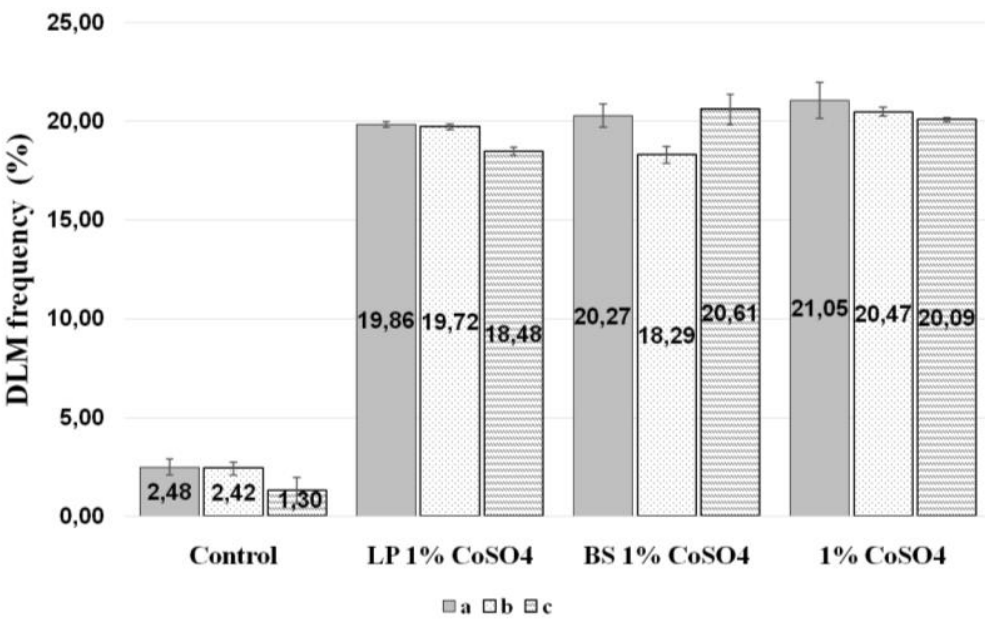

Fig. 5. Dynamics of death of F1 offspring at different stages of ontogenesis 


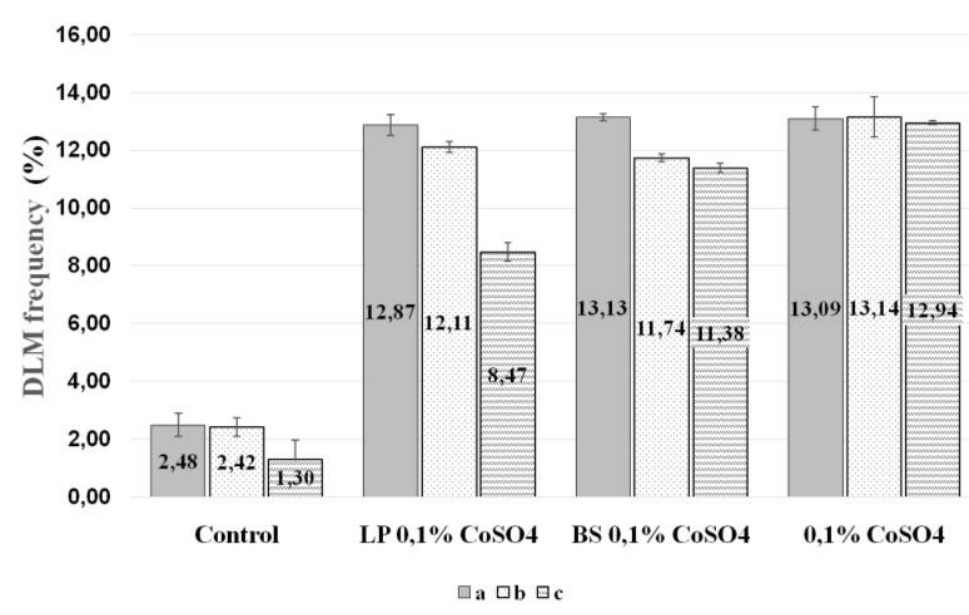

Fig. 6. Dynamics of death of F1 offspring at different stages of ontogenesis

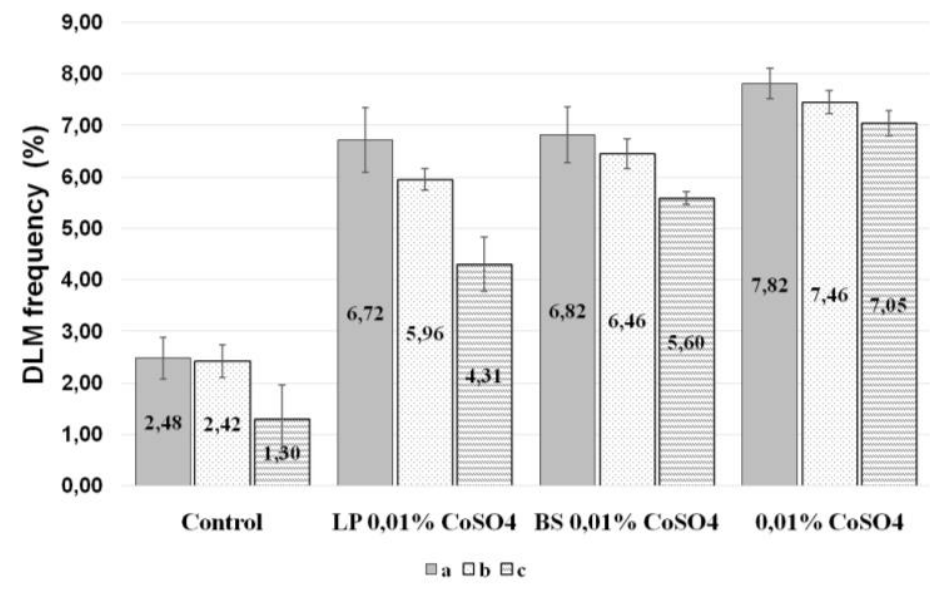

Fig. 7. Dynamics of death of F1 offspring at different stages of ontogenesis

In all experimental groups with a $1 \%$ concentration of cobalt sulfate, there is no significant decrease in the percentage of dominant lethal mutations after preliminary keeping of males on nutrient media with probiotics, except for one experimental group with a probiotic preparation based on L. plantarum culture with an exposure period of 7 days.

A preliminary exposure of Drosophila males on a medium with a probiotic preparation based on $B$. subtilis for 3 days significantly reduces the percentage of DLM in samples with a heavy metal salt concentration of $0.1 \%$ and for 7 days in samples with a heavy metal salt concentration of $0.1 \%$ and $0.01 \%$.

The preliminary exposure of Drosophila males on a medium with a probiotic preparation based on L. plantarum for 3 days and 7 days significantly reduces the percentage of DLM in samples with a heavy metal salt concentration of $0.1 \%$ and $0.01 \%$.

The data obtained indicate the possibility of using the prepared probiotic preparations as antimutagens in relation to low concentrations of cobalt sulfate. At the same time, the longer the time of keeping Drosophila on a nutrient medium with probiotics, the stronger the positive effect of the drug is. The lack of antimutagenic activity of $B$. subtilis and $L$. plantarum in relation to $1 \%$ concentration of $\mathrm{CoSO}_{4}$ requires additional verification with a longer exposure time of males. This can lead to a positive result in this experience. 


\section{References}

1. Semina N.A.T.4 (2004)

2. Feoktistova, N.V. Scientific notes of Kazan University. Series Natural Sciences. 1, 159 (2017)

3. Belokon E.M. Guidelines for the determination of the mutagenicactivity of chemicalpreparations on Drosophila (Lviv, Leningrad State University, 1984) 